\title{
Simultaneous pulmonary and intra- thoracic lymph nodal granulomatosis of unknown significance (GLUS)
}

\author{
S. Katsenos ${ }^{1}$, K. Kostikas ${ }^{1}$, S. Lachanis², D. Sabaziotis³, \\ K. Psathakis ${ }^{1}$, S. Loukides 1
}

ABSTRACT: Simultaneous pulmonary and intra-thoracic lymph nodal granulomatosis of unknown significance (GLUS). S. Katsenos, K. Kostikas, S. Lachanis, D. Sabaziotis, K. Psathakis, S. Loukides.

A case of a 30-year-old male with a fever, dry cough and associated abnormal findings in imaging modalities (bilateral hilar lymphadenopathy and nodular parenchymal opacities) is described.
After a further and scrutinized work-up, the diagnosis of GLUS syndrome was made. Clinical, etiological, pathological and therapeutical aspects of the disease are discussed, demonstrating the paramount importance of the use of the immunohistochemical methods in the diagnosis of this disorder.

Monaldi Arch Chest Dis 2004; 61: 2, 120-123.

Keywords: Fever, bilateral hilar lymphadenopathy, nodular opacities, GLUS.

1 Department of Pneumonology, 401 General Army Hospital of Athens, Athens, Greece.

2 Department of Radiology, 401 General Army Hospital of Athens, Athens, Greece.

3 Department of Pathology, 401 General Army Hospital of Athens, Athens, Greece.

Correspondence: Stelios Loukides, MD; 2, Smolika St; 16673 Voula, Athens, Greece; e-mail: ssat@hol.gr

\section{Case report}

A 30 years old male was referred to the authors' department with complaints of a fever $\left(39,5^{\circ} \mathrm{C}\right)$, non-productive cough, myalgias and fatigue for 10 days before his admission to the hospital. Upon admission, a chest radiograph was performed showing bilateral hilar lymphadenopathy accompanied by fluffy nodular and micronodular opacities predominating in the middle lung fields (figure 1). The patient received one course of antibiotics for common bacterial pathogens, before he sought our medical assistance, with no remission of the above-mentioned symptoms. He was an active smoker (10 pack/years). He worked as an employee in an office in a private company. $\mathrm{He}$ had been in good health previously.

A thorough physical examination and laboratory evaluation at the time of the admission was normal. Briefly, there were no abnormal findings from the examination of the respiratory system, and there were no signs of involvement of peripheral lymph nodes, hepatic disease or skin lesions. Complete blood count and standard serum biochemistry tests were normal. Serum angiotensin converting enzyme (SACE) was within normal limits (55 U/L). Serum and urine calcium levels were normal. Laboratory tests for collagen-vascular diseases (i.e. $\mathrm{C} 3$ and $\mathrm{C} 4$ complement components, antinuclear antibodies, c and p ANCA), as well as erythrocyte sedimentation rate (ESR) and C-reactive protein
(CRP) were normal. Serologic tests for EbsteinBarr virus (EBV), cytomegalovirus (CMV) and Toxoplasma and Brucella were negative. Additionally, serologic tests for hepatitis A, B and C, as well as for human immunodeficiency virus were negative. Arterial blood gases were normal. Electrocardiogram and echocardiogram were normal, excluding any heart involvement. An ultrasonographic ex-

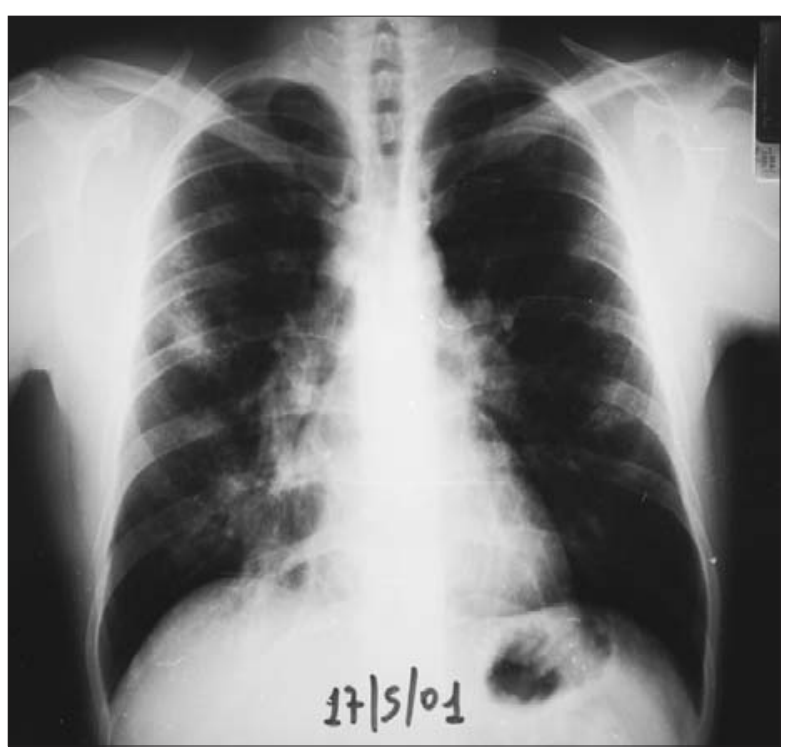

Fig. 1. - Chest-X-ray of the patient showing bilateral hilar lymphodrenopathy accompanied by fluffy nodular and micronodular opacities predominating in the middle lung fields. 
amination of the upper abdomen did not reveal any liver or spleen lesions. Skin tests for both M. tuberculosis and atypical mycobacteria were negative.

Further imaging evaluation with high-resolution computed chest tomography (CT) disclosed symmetrical bilateral and mediastinal lymphadenopathy as well as confluent interstitial micronodules forming larger nodular lesions up to 2 $\mathrm{cm}$ in size with a predilection in the middle zone of the lung (figure 2).

In the context of the investigation, the patient underwent fiberoptic bronchoscopy without endobronchial lesions, but only a slight mucosal redness in the whole tracheobronchial tree was observed. Bronchoalveolar lavage (BAL) samples for mycobacteria and other common pathogens as well as BAL cell differential counts, transbronchial biopsies and transbronchial needle aspiration (TBNA) from anterior subcarinal lymph nodes did not reveal remarkable abnormal findings.

Because the patient continued to have fever, we referred him to the thoracic surgery department where an open lung biopsy was performed. Specimens were obtained both by lung parenchyma and mediastinal lymph nodes. The histopathology (fig-

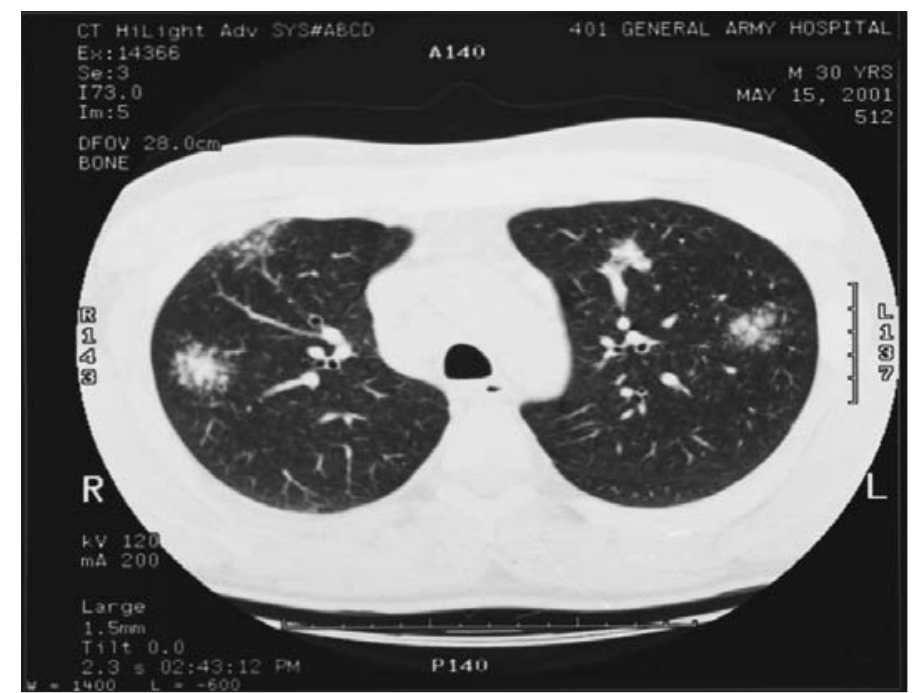

Fig. 2. - Computed chest tomography showing symmetrical bilateral and mediastinal lynphoadenopathy as well as confluent interstitial micronodules forming larger nodular lesions up to $2 \mathrm{~cm}$ in size with a predilection in the middle zone of the lung.

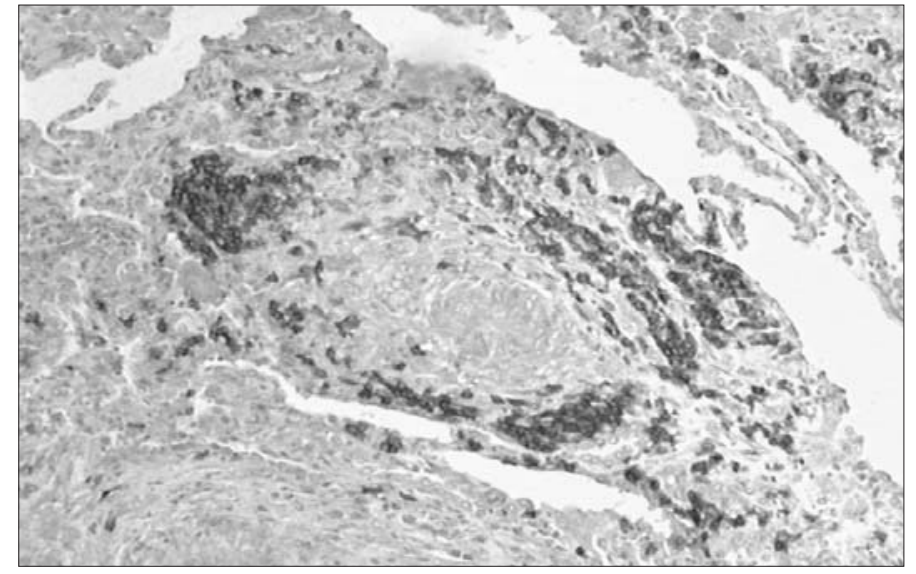

Fig. 3. - Immunohistochemical staining of the lung biopsy specimen with antiCD20 monoclonal antibody. ure 3) showed marked occupation of the lung and lymph node section by abundant, confluent and small sized granulomas. These granulomas consisted of epithelioid histiocytes and a small number of lymphocytes, plasma cells and sparsely located eosinophils. Several polynuclear giant cells (Langhans cells) were also noted. There were typical asteroid bodies as well as scarce Schauman bodies in the giant cells' cytoplasm. Microvesicular degeneration with small granules, which were stained negatively with fungi dyes, was visible in the cytoplasm of a few polynuclear giant cells. Non-caseating foci of necrosis with a little cellular debris were seen in a small portion of granulomas as well as foci of hyaline necrosis, which were slightly larger sized, shapeless and encompassed by foamy histiocytes. Focal fibrotic and sclerotic changes developed among the granulomas. Some granulomas surrounded bronchioles and small bronchi without destruction of their wall. Bronchi presented slight dilation and were filled with mucus, foamy histiocytes, inflammatory elements and exfoliative epithelia. Distinctive features of vasculitis were not found. The polymerase chain reaction (PCR) and culture of this specimen for TBC (tuberculosis) were negative. The lymphocytes surrounding the granulomas stained positively to a large extent with B-cell markers (CD20, CD45RA) by immunohistochemical analysis. A few CD45Ro T cells were also present. In conclusion, a granulomatous reaction was observed with the presence of epithelioid (sarcoid form) granulomas and small foci of necrosis.

A diagnosis of simultaneous pulmonary and intra-thoracic lymph nodal granulomatosis of unknown significance (GLUS) was made.

We administered corticosteroids (40 mg prednisolone/day with gradual tapering) in our patient, who responded satisfactorily in this regimen in terms of clinical picture and imaging features. The patient is in the best of health and is re-evaluated at regular intervals. After 3 months from the end of treatment the disease has been completely regressed, as it is proven by the total resolution of the pulmonary infiltrates on a new CT scan.

\section{Discussion}

Most often, it is possible to clarify the etiology of granulomatous lesions with reasonable confidence, but there is a residual group of unclassifiable cases in which the presence of the granulomas remains unexplained in spite of all relevant microbiological, biochemical, serological, histopathological studies and further work-up. It has been determined that the percentage of such cases fluctuates between $15-20 \%$ [1]. This category of undetermined granulomatous lesions, which is characterised by a prolonged fever, involvement of liver, lymph nodes, lung, bone marrow, spleen, a benign course and a ten- 
dency to recurrence, has been designated as the GLUS (granulomatous lesions of unknown significance) syndrome by Brincker and co-workers [2].

In summary, evidence from several sources confirms the existence of a febrile, granulomatous, multisystem disease with moderate abnormalities of liver function, constitutional symptoms, hypergammaglobulinemia, responsive to immunosuppressive therapy and splenectomy and a propitious prognosis. Hepatic symptoms predominate in some patients whereas others present with regional lymphadenopathy, splenic symptoms or prolonged unexplained fever. A subacute course was observed in some cases. All of the above clinical features except prolonged fever were not noticed in our case, emphasizing that their presence is related to the site of disease involvement.

For the time being, a diagnosis of the GLUS syndrome should be considered in patients in whom a biopsy from one or more tissues has shown the presence of epithelioid cell granulomas (with or without necrosis) that cannot be accounted for. Clearly, a diagnosis of this strange pathologic entity should not be made unless all the recognized causes of granulomatous lesions have been excluded by appropriate microbiological, biochemical, serological and histopathological tests. It is important to follow-up such patients, especially those who present with fever as a main clinical manifestation, since the course may be prolonged with intermittent signs of disease activity. Thus, all the conditions which can cause pulmonary granulomas have been listed in the differential diagnosis of our case (table 1), but there was no clinical or laboratory evidence to support any of these [3]. Specifically, serological tests for other infectious agents known to cause granuloma formation, such as EBV, CMV, Toxoplasma gondii, Brucella, or even hepatitis viruses, proved to be negative in our patient. Parasitic infections (schistosomiasis, leishmaniasis) were excluded due to the absence of their characteristic clinical and laboratory manifestations. The diagnoses of fungal diseases were ruled out by the negative BAL cultures. Immunological studies excluded the presence of pulmonary vasculitides. The aforementioned histological examination did not present findings suggestive of malignancies and lymphoproliferative disorders affecting the lung. Hypersensitivity pneumonitis, berylliosis and other occupational potentially granulomatous diseases were also excluded because our patient did not fulfill neither the diagnostic criteria nor the environmental and occupational exposure history.

Nevertheless, the particular relationship between sarcoidosis and GLUS syndrome is worth mentioning. Although the combination of clinical features described above matches that of sarcoidosis, it is distinguished from the latter by the absence of hypercalcemia and a high titer of SACE. In contrast to sarcoidosis, there is no doubt that granulomas with necrotic lesions are present in cases of GLUS, irrespective of location. Using immunohistochemical methods, it has been demonstrated that granulomatous lesions can be separated into two different families of cases according to the presence or absence of intragranulomatous B lymphocytes [4]. "Old, chronic" granulomas associated with a high rate of fibrosis and a high rate of transformation of macrophages into giant cells are B-cell negative. This category includes sarcoidosis and mycobacterial infection. The B-cell positive family of "young, inflammatory" granulomas is associated with sinus histiocytosis and consists of GLUS, tumor-related sarcoid reactions, toxoplasmosis and Crohn's disease. Figure 3 illustrates clearly the difference between sarcoidosis and GLUS. It has been also observed that the granulomas of GLUS contain natural killer (NK) cells along with the B lymphocytes, whereas NK cells do not appear in the granulomas of sarcoidosis [5]. In summary, although granulomatous lesions due to various etiologies may have similar appearances, both histomorphological and immunohistological differences can be demonstrated, particularly between the granulomas of sarcoidosis and GLUS, supporting the clinical evidence that the GLUS syndrome is distinguishable from sarcoidosis.

In the past, a few cases of patients with unexplained granulomatous lesions, either in lung tissue biopsies [6] or in peripheral lymph nodes [7] have been reported. To the best of the authors' knowledge, this is the first report of GLUS syndrome located simultaneously both in the lung parenchyma and the intrathoracic lymph nodes.

Generally, the prognosis of GLUS syndrome appears to be excellent, with spontaneous resolution in most cases. In others, intermittent signs of

Table 1. - Differential diagnosis of pulmonary granulomatosis\#

1) Infections [tuberculosis, fungal (e.g. aspergillosis, histoplasmosis), bacterial (e.g. brucellosis), parasitic (schistosomiasis, leishmaniasis, toxoplasmosis), viral (Epstein-Barr virus, Cytomegalovirus, Hepatitis viruses)]

2) Malignancies and lymphoproliferative disorders [primary pulmonary lymphoma, mucosa associated lymphoid tissue (MALT) type NHL, lymphocytic interstitial pneumonia, lymphomatoid granulomatosis]

3) Diseases of unknown etiology [sarcoidosis, Wegener's granulomatosis, Churg-Strauss syndrome, bronchocentric granulomatosis, pulmonary Langerhans' cell histiocytosis (eosinophilic granuloma)]

4) Environmental or occupational disorders (hypersensitivity pneumonitis, berylliosis, silicosis, talcosis)

\#: for more details see reference [3]. 
disease activity, especially in cases with febrile presentations, may be present for months or even years. In such cases, we prefer the administration of corticosteroids, which constitute the responsive therapy $[8,9]$. Regression of disease has been also reported by cytostatic treatment [9]. In corroboration of the satisfactory corticosteroid action was the fact that our patient, who received this medication form, improved his clinical condition.

In conclusion, the GLUS syndrome is diagnosed by exclusion of other causes of granulomatous lesions. It should always be borne in mind, irrespective of its frequency, in the differential diagnosis of pulmonary granulomas. We must highlight the value of the immunohistochemical method, which is very helpful in the discrimination among the granulomatous lesions and particularly between the granulomas of sarcoidosis and GLUS. The demonstration of the presence of $\mathrm{B}$ lymphocytes and natural killer cells within the granulomas favours the diagnosis of GLUS syndrome. The most definitive method of establishing the diagnosis is surgical (open) lung biopsy, because it provides a larger quantity of tissue. Besides the adequate specimen, a skilled pathologist is essential for the diagnosis.

\section{References}

1. American Thoracic Society: statement on sarcoidosis. Am J Respir Crit Care Med 1999; 160: 736-755.

2. Brincker H. Granulomatous lesions of unknown significance in biopsies from lymph nodes and other tissues: the GLUS syndrome. Sarcoidosis 1990; 7: 28-30.

3. Sharma OP, Flora G. Diagnosis of pulmonary granulomas in the tropics. Semin Respir Med 1991; 12: 124135.

4. Brincker H, Pedersen NT. Immunological marker patterns in granulomatous lymph node lesions. Histopathology 1989; 15: 495-503.

5. Brincker H, Pedersen NT. Immunohistologic separation of B-cell positive granulomas from B-cell negative granulomas in paraffin-embedded tissues with special reference to tumor-related sarcoid reactions. APMIS 1991a; 99: 282-290.

6. Segal EL, Starr GF, Weed LA. Study of surgically excised pulmonary granulomas. JAMA 1959; 170: 515522.

7. Schroder KE, Elverland HH, Mair IWS, Liavaag PG. Granulomatous cervical lymphadenitis. J Otolaryngol 1979; 8: 127-131.

8. Friedland JS, Weatherall DJ, Ledingham JGG. A chronic granulomatous syndrome of unknown origin. Medicine 1990; 69: 325-331.

9. Telenti A, Hermans PE. Idiopathic granulomatosis manifesting as fever of unknown origin. Mayo Clin Proc 1989; 64: 44-50.

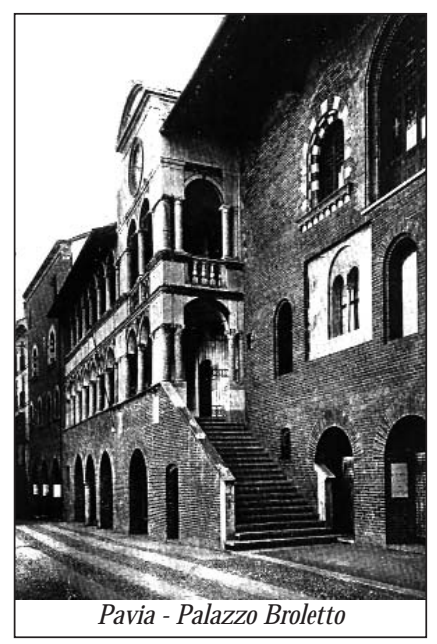

